\title{
Assessment of Linearity Improvement in Optical Communication Systems with Machine Learning Methods
}

\author{
Muhammad Usman Hadi ${ }^{1}$ \\ ${ }^{1}$ Department of Electronic and Information Engineering, University of Bologna, Italy.
}

Corresponding author: M. U. Hadi;

email: usmanhadi@ieee.org

Use of Machine Learning (ML) methodologies in optical communications has paved a new pathway. In this paper, firstly, we discuss the use of ML methodologies for reducing optical fiber nonlinearities, nonlinearity compensation, fault detection and optical performance monitoring. Then we present our recent work where we compare RL-SARSA and SVM based method with conventional method. The results show that RL-SARSA and SVM methods are successful candidates in mitigating the nonlinearities in proposed system as compared to conventional optical communication system.

Keywords: radio over fiber; nonlinearities mitigation; support vector machine method; RLSARSA

\section{INTRODUCTION}

Radio over Fiber (RoF) technology has been realized in different forms ranging from analog to more complex forms [1-6]. The enhancement of capacity and wireless coverage has posed significant challenges to the existing optical and wireless access networks. Machine learning (ML) methods have given a new direction to meet the ever-increasing challenges in fiber-optic communications. Since, ML-based methods are well known to perform exceptionally well in scenarios where it is too difficult to explicitly describe the underlying physics and mathematics of the problem and the numerical procedures available require significant computational resources/time.

Before the introduction of ML in this field, the reduction of nonlinearities was carried out through different methodologies that were proposed during last 10 years via Optical and Electrical domain methods, with latter getting more attention. Digital Predistortion has gained immense attention and therefore it is still looked to be an important methodology [1,7-9]. However, these DPD based linearization techniques are not so straight forward and are rather complex, time consuming and adds extra over heads [10]. Similarly, these methodologies require an extra feedback mechanism which is achievable but increases the complexity of the system. Therefore, reducing such nonlinearities in optical communication links effectively is still a bottle neck in efficient deployment of optical communication systems. 
Recent advancements in ML technology have further motivated the researchers to explore true potential of this emerging field in optical communication systems. Firstly, we review some significant research ideas pertaining to the use of ML algorithms in fiber-optic communications. Then, a experimental study is presented for the mitigation of nonlinear impairments by comparing Reinforcement Learning (RL) based machine learning method with Support Vector Machine (SVM) method and conventional methods. In the proposed system, 10-Gb/s with 256 quadrature amplitude modulation (QAM) signal is injected into distributed feedback (DFB) laser for $50 \mathrm{~km}$ single mode fiber transmission. It causes signal to suffer from nonlinearities due to opto-electronic devices in the RoF link as the ideal decision boundary is no longer linear.

\title{
II. Machine Learning Applications in Optical Communication System
}

The application of optical communication systems can be divided with three main subcategories:

\author{
i. Nonlinearity Mitigation \\ ii. Performance Monitoring \\ iii. Smart Decision/ Prevention
}

The mitigation of nonlinearities in optical communications with ML is the most important application these days. Recently, RL-SARSA based ML method has been evaluated in [11-12] while SVM and KNN based ML methods have been utilized too in [13-15]. SBP method has also been evaluated for reducing the impairments in optical communication systems [16].

The other important application of ML is monitoring the performance of optical communication system. Many methodologies have been evaluated among which SVM [17], ANN [18] and Kernel based methods are most significant [19]. Recently, Parzen window method to mitigate the fiber nonlinearity in the context of dispersion managed and dispersion unmanaged systems has been proposed as well [20].

Finally, the other important application of ML method is smart decision in order to prevent any errors by fault detection. Reliable operation of an optical network requires incorporation of an early protection mechanism into the network. Recently, several ML-based techniques have been developed for cognitive fault detection/prevention in fiber-optic networks. A combination of double exponential smoothing (DES) and SVM for network equipment failure prediction has been employed in [20]. In [17,21-22], an ANN is trained to learn historical fault patterns in networks and is subsequently used for detecting significant network faults with much better accuracies and proactive reaction times as compared to traditional threshold-based fault detection methods. Figure 1 presents the summary of the applications of ML methods in optical communication systems. 


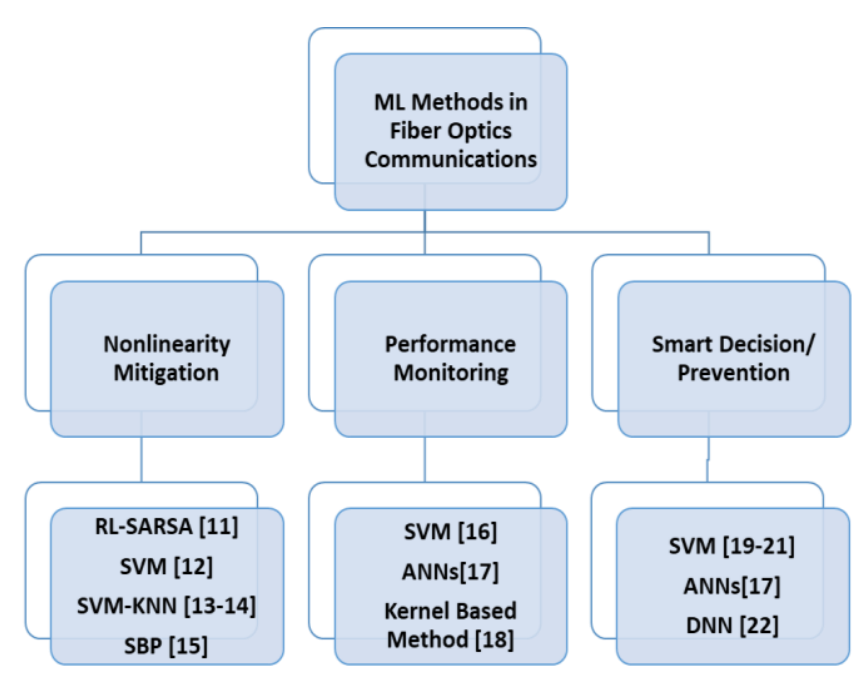

Fig. 1: Key applications of ML methods in optical fiber systems

\section{Experimental Setup}

RL-SARSA method [13] and SVM methods [11] have been separately studied recently however, the comparison together with conventional method has not been evaluated. The experimental setup utilized is demonstrated in Fig. 2. Distributed Feedback (DFB) laser is driven by arbitrary waveform generator (AWG) operating at $10 \mathrm{Gbits} / \mathrm{s}$. The modulated 256QAM signal is used while the laser diode (LD) is working at $1550 \mathrm{~nm}$. The Single Mode Fiber (SMF) of $50 \mathrm{~km}$ length is followed by variable optical attenuator referred as VOA whose functionality is to adjust the received optical power. The signal is photo detected by a photodiode with $0.7 \mathrm{~A} / \mathrm{W}$ of responsivity. Firstly, the signal received is sent to parameter estimation lock without the compensation through the ML block.

Then, the signal is passed through the digital processing block for machine learning decisions in offline manner. Here, the RL-SARSA and SVM methods are utilized that finds the optimum decision boundary. Minimal optimization algorithm is used to find the boundary condition while the training threshold is at about $5.1 \%$ with 1400 symbols to automatically obtain the optimal parameters. Then, ML compensated results are compared with conventional results.

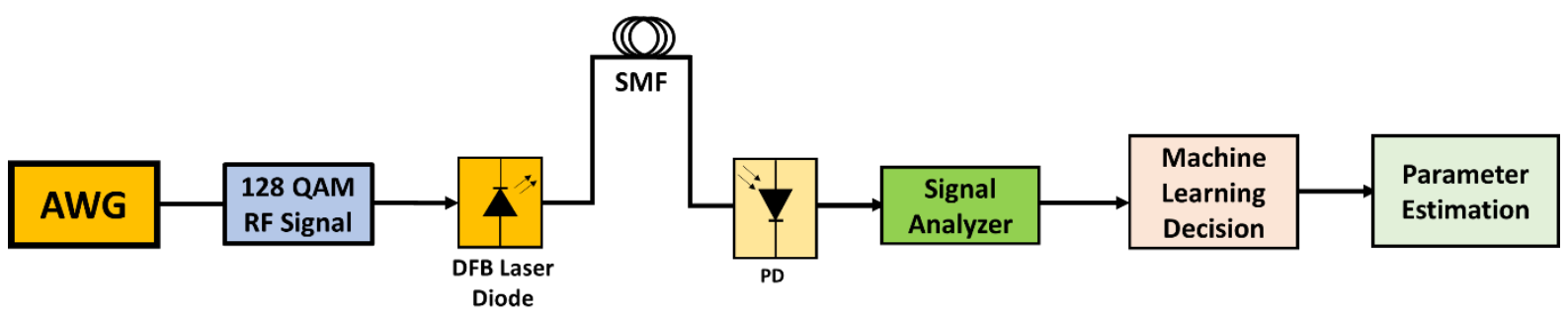

Fig. 2. Experimental setup. AWG: Arbitrary Waveform Generator, DFB laser: Distributed Feedback laser, VOA: Variable Optical Attenuator, SMF: Single Mode Fiber 


\section{Experimental Results and Discussion}

The experimental results are discussed in this section. SVM and RL-SARSA based ML method is compared with conventional decision method in. Fig. 3 with respect to number of training data points for BER. It is clear that BER is decreased with ML methods. Indeed, after 700 training data points, SVM outperforms RL-SARSA method too.

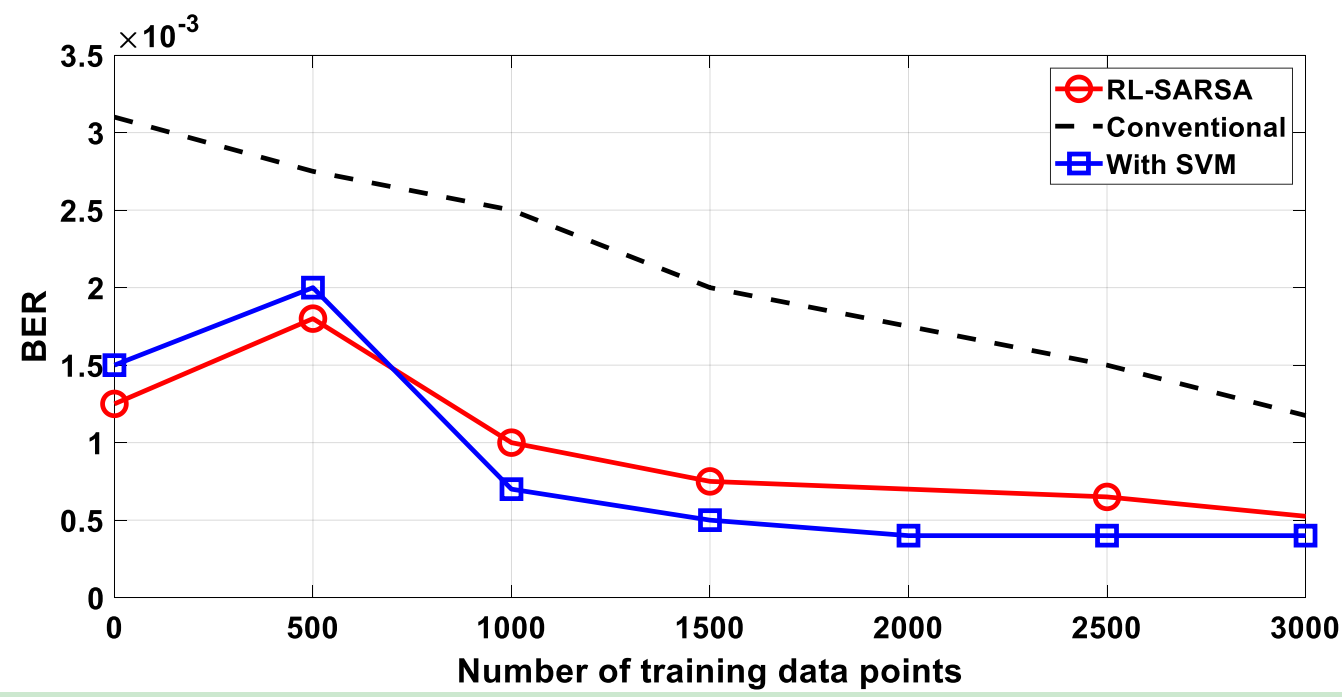

Fig. 3. BER vs number of training data points with respect to RL-SARSA, With SVM and Conventional method

Then, In Fig. 4, BER is compared with changing input power is compared for RL-SARSA, SVM and conventional method. It can be seen that with increasing RF input power, the ML guided decision results in better reduction in nonlinearities as compared to conventional decision method. Both ML methods, RL-SARSA and SVM results in same correction.

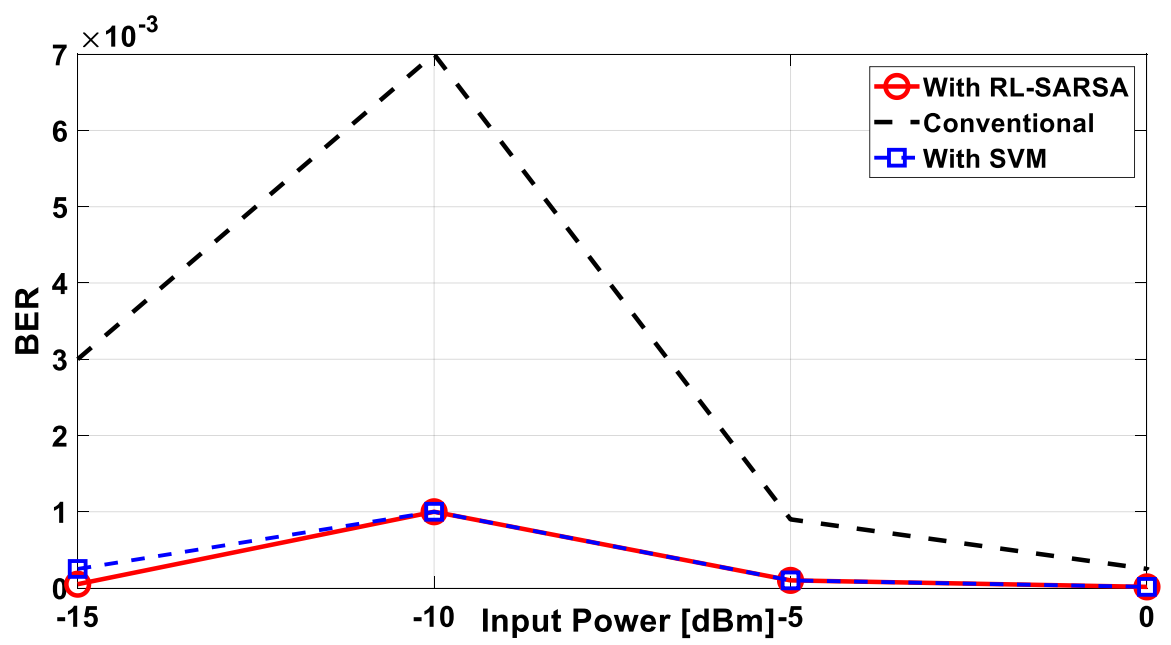

Fig. 4. BER vs RF input power with respect to RL-SARSA, With SVM and Conventional method 
Similarly, a received optical power is evaluated with respect to BER for ML methods and noncompensated one is compared in Fig. 5. The received optical power has lesser BER with RLSARSA as compared to SVM method. Indeed, SVM has good reduction in BER but with RLSARSA, a better results are achieved.

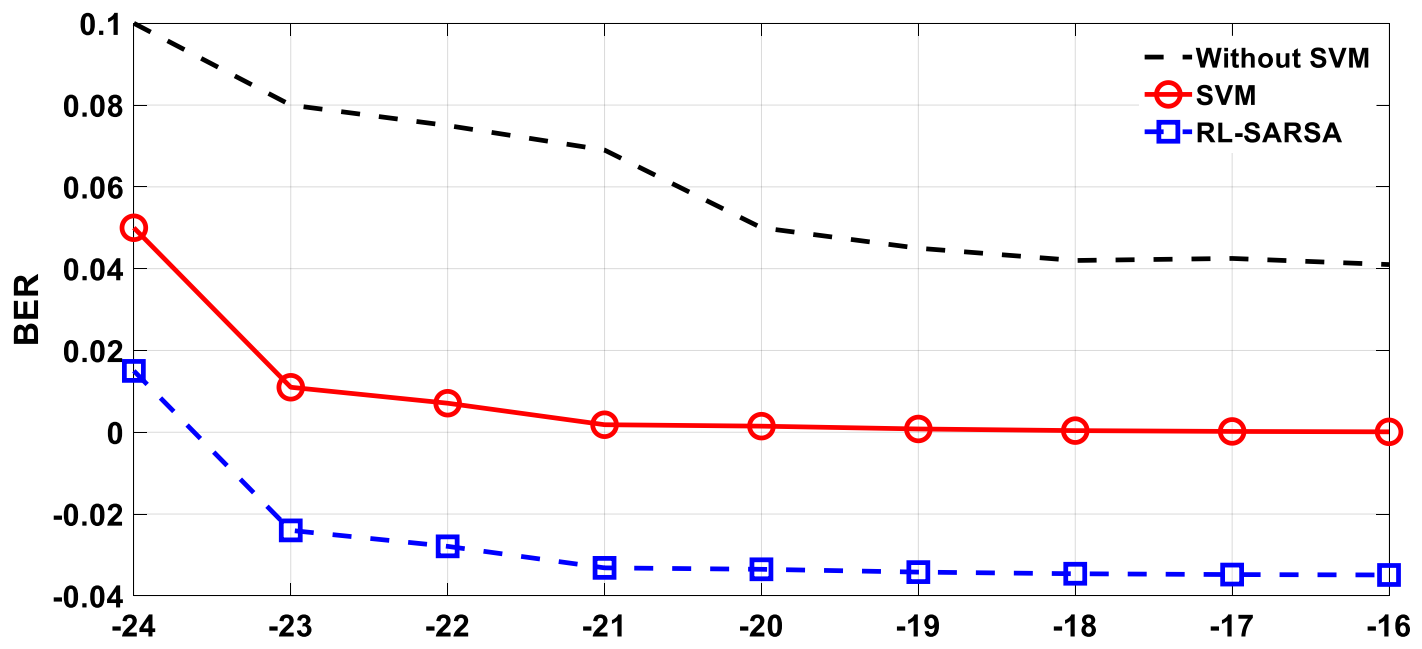

Fig. 5. BER vs received optical power with respect to RL-SARSA, With SVM and Conventional method

\section{CONCLUSION}

The article provides a recent applications of ML methods in optical communication systems. The survey presents the categories of applications where ML has been successfully being employed. Then, RL-SARSA and SVM are employed in our recent work and results are compared with respect to conventional uncompensated results. Indeed, the ML methods proposed are quite useful in mitigating the nonlinearities of optical communication systems.

\section{REFERENCES}

[1] Hadi, M.U., Nanni, J., Polleux, JL. et al. Opt Quant Electron (2019) 51: 205. https://doi.org/10.1007/s11082-019-1923-8

[2] Muhammad Usman Hadi, Hyun Jung, Salman Ghaffar, Pier Andrea Traverso, Giovanni Tartarini, Optimized digital radio over fiber system for medium range communication, Optics Communications, Volume 443, 2019, Pages 177-185, ISSN 0030-4018, https://doi.org/10.1016/j.optcom.2019.03.037.

[3] Hadi, M., Jung, H., Traverso, P., et al. (2019). Digital Radio Frequency Transport over Optical Fiber for 5G Fronthaul Links. Journal of Optical Communications, doi:10.1515/joc2019-0051

[4] Hadi M, Aslam N, Jung H. (2019). Performance appraisal of sigma delta modulated radio over fiber system. Journal of Optical Communications, doi:10.1515/joc-2018-0227. 
[5] Hadi, M., Hadi, M., Aslam, N., et al. (2019). Experimental Demonstration of MASH Based Sigma Delta Radio over Fiber System for 5G C-RAN Downlink. Journal of Optical Communications, doi:10.1515/joc-2019-0011

[6] Muhammad Usman Hadi, Pier Andrea Traverso, Giovanni Tartarini, Hyun Jung, Experimental characterization of Sigma Delta Radio over fiber system for 5G C-RAN downlink, ICT Express, 2019, ISSN 2405-9595, https://doi.org/10.1016/j.icte.2019.06.002.

[7] M. U. Hadi, "Extending the benefits of LTE to unlicensed spectrum," 2015 International Conference on Information and Communication Technologies (ICICT), Karachi, 2015, pp. 13. doi: 10.1109/ICICT.2015.7469592

[8] M. U. Hadi et al., "Experimental evaluation of digital predistortion for VCSEL-SSMFbased Radio-over-Fiber link," 2018 International Topical Meeting on Microwave Photonics (MWP), Toulouse, 2018, pp. 1-4.doi: 10.1109/MWP.2018.8552895

[9] M. U. Hadi, P. A. Traverso, G. Tartarini, O. Venard, G. Baudoin and J. Polleux, "Digital Predistortion for Linearity Improvement of VCSEL-SSMF-Based Radio-Over-Fiber Links," in IEEE Microwave and Wireless Components Letters. doi:

10.1109/LMWC.2018.2889004

[10] Hadi, M.U., Nanni, J., Traverso, P.A., Tartarini, G., Venard, O., Baudoin, G., Polleux, J.L.: Linearity improvement of VCSELs based radio over fiber systems utilizing digital predistortion. Adv. Sci. Technol. Eng. Syst. J. 4(3), 156-163 (2019). doi: 10.25046/aj040321 [11] Hadi, M.U. RL-SARSA Machine Learning Based Analog Radio over Fiber System. Preprints 2019, 2019090159 (doi: 10.20944/preprints201909.0159.v1

[12] N. Aslam, K. Xia and M. U. Hadi, "Optimal Wireless Charging Inclusive of Intellectual Routing Based on SARSA Learning in Renewable Wireless Sensor Networks," in IEEE Sensors Journal, vol. 19, no. 18, pp. 8340-8351, 15 Sept.15, 2019. doi:

10.1109/JSEN.2019.2918865

[13] Hadi, M.U. Nonlinearities Diminution in 40 Gb/s 256 QAM Radio over Fiber Link via Machine Learning Method. Preprints 2019, 2019090031 (doi:

10.20944/preprints201909.0031.v1).

[14] M. Li, S. Yu, J. Yang, Z. Chen, Y. Han, and W. Gu, "Nonparameter nonlinear phase noise mitigation by using M-ary support vector machine for coherent optical systems," IEEE Photonics Journal, vol. 5, no. 6, 2013.

[15] Z. Wang et al., "Failure prediction using machine learning and time series in optical network," Opt. Exp., vol. 25, no. 16, 2017.

[16] N. Jiang et al., "Stochastic backpropagation for coherent optical communications," in Proc. ECOC, Geneva, 2011, Paper We.10.P1.81

[17] R. A. Skoog et al., "Automatic identification of impairments using support vector machine pattern classification on eye diagrams," IEEE Photon. Technol. Lett., vol. 18, no. 22, pp. 2398-2400, 2006.

[18] F. N. Khan, Y. Yu, M. C. Tan, C. Yu, A. P. T. Lau, and C. Lu, "Simultaneous OSNR monitoring and modulation format identification using asynchronous single channel sampling," in Proc. Asia Communications and Photonics Conf., Hong Kong, 2015, Paper AS4F.6.

[19] T. B. Anderson et al., "Multi impairment monitoring for optical networks," J.

Lightwave Technol., vol. 27, no. 16, pp. 3729-3736, 2009. 
[20] A. Amari, X. Lin, O. A. Dobre, R. Venkatesan and A. Alvarado, "A Machine LearningBased Detection Technique for Optical Fiber Nonlinearity Mitigation," in IEEE Photonics Technology Letters, vol. 31, no. 8, pp. 627-630, 15 April15, 2019.

doi: 10.1109/LPT.2019.2902973

[21] D. Rafique et al., "Cognitive assurance architecture for optical network fault management, J. Lightwave Technol., vol. 36, no. 7, pp. 1443-1450, 2018.

[22] Aslam, N.; Xia, K.; Haider, M.T.; Hadi, M.U. Energy-Aware Adaptive Weighted Grid Clustering Algorithm for Renewable Wireless Sensor Networks. Future Internet 2017, 9, 54. 